\title{
Biological effects of FoxJ2 over-expression
}

\author{
F. Martín-de-Lara $\cdot$ P. Sánchez-Aparicio $\cdot$ \\ Carmen Arias de la Fuente · J. Rey-Campos
}

Received: 31 March 2008/Accepted: 28 July 2008/Published online: 26 August 2008

(C) The Author(s) 2008. This article is published with open access at Springerlink.com

\begin{abstract}
As reported previously, we have extensively studied FoxJ2, a member of the Fork Head transcription factors family. While the biochemical and functional structures of this transcription factor are well understood, its biological function remains unknown. Here, we present data that address this point using transgenic mouse technology. We found that the birth rate and the number of transgenic animals obtained when transferring embryos over-expressing the FoxJ2 protein were lower than those obtained with embryos over-expressing a control protein, suggesting FoxJ2 overexpression has a negative effect on embryonic development. Transient Fox 2 transgenesis experiments have confirmed that FoxJ2 over-expression has a lethal effect on embryonic development from E10.5. Moreover, in vitro culture of FoxJ2-microinjected embryos demonstrated a significant developmental blockage, indicating that FoxJ2 could also have an effect on preimplantation stages. Most probably, these negative
\end{abstract}

F. Martín-de-Lara · P. Sánchez-Aparicio ·

C. Arias de la Fuente $\cdot$ J. Rey-Campos ( $\square)$

Centro de Investigaciones Biológicas, CSIC,

Madrid, Spain

e-mail: Javier.rey@orgc.csic.es

F. Martín-de-Lara · J. Rey-Campos

CSIC, Madrid, Spain

e-mail: fmlara@orgc.csic.es

P. Sánchez-Aparicio

Clínica de Medicina de la Reproducción y Ginecología,

FivMadrid, Madrid, Spain effects of FoxJ2 over-expression during development also explain the low percentage of adult transgenic mice obtained. Furthermore, most of the transgenic mice that lived to adulthood did not show transgene expression. In fact, the only two adult transgenic animals (one male and one female) in which FoxJ2 transgene expression was detected showed a mosaic expression and died prematurely as a result of cardio-respiratory failure. Postmortem analysis of these animals revealed a hypertrophic heart and abnormal testes in the male. In order to identify genes regulated by Fox 2 consistent with the phenotypes observed for FoxJ2 transgenic mice, EMSA assays and co-transfection experiments were carried out. Our data indicate that the genes coding for the gap junction protein Connexin-43 and the cellcell contact protein E-Cadherin, may be good candidates for FoxJ2-regulated genes. Interestingly, Connexin-43 and E-Cadherin show expression patterns similar to FoxJ2, and the phenotypes of Connexin-43 and E-Cadherin mutants resemble those of our FoxJ2 transgenic animals. These data suggest that the lethal effect on embryonic development of FoxJ2 overexpression, as well as the alterations observed in the heart and testes of adult transgenic mice, could be determined by changes in the transcription of genes such as Connexin43 and/or E-Cadherin.

Keywords FoxJ2 - Transgenic mice · Development · Fork Head · Transcription factors · Cad E · Connexin 43 - Fertility · Heart hypertrophy · Infertility 


\section{Introduction}

FoxJ2 is a member of the Fork Head family of transcription factors (Pérez-Sánchez et al. 2000a). All family members, of which there are more than 80 , share a highly conserved DNA-binding domain called the Fork-Head motif (Kaufmann and Knochel 1996; Granadino et al. 2000a) which is a variant of the helix-turn-helix motif firstly identified in Drosophila (Weigel et al. 1989).

Most members of the Fork Head family have been reported to be widely distributed across several organs and tissues in very different species, from yeast to humans. However, some Fork Head factors exhibit remarkably constrained patterns in particular cases, such as at particular moments in development (Choi et al. 2006). The role played by these transcription factors is important for various biological processes such as signal transduction (Tan et al. 1998; Zhou et al. 1998), cell differentiation (Kaestner et al. 1997; Kume et al. 1998), or even controlling longevity (Lin et al. 1997; Ogg et al. 1997).

FoxJ2 is found in several mammals and other vertebrates (Pérez-Sánchez et al. 2000b; Choi et al. 2006; Wijchers et al. 2006), and is widely distributed in different organs and tissues not only in adults, but also in the fetus. The broad pattern of expression in adults includes gonads (ovaries and testes), among other organs (Pérez-Sánchez et al. 2000a, b). The expression of FoxJ2 is activated during spermatogenesis, from pachytene spermatocytes to round spermatids, although not in spermatogonia. In addition to the germ lineage, testicular Sertoli cells also showed expression of FoxJ2. By contrast, in the ovaries, only follicular granulosa cells expressed FoxJ2. Neither oocytes nor mature spermatozoa have shown FoxJ2 expression (Granadino et al. 2000a, b). FoxJ2 starts to be expressed early in embryonic development: blastocyst (trophectoderm and inner cell mass) and even 8-cell stage embryos express this transcription factor (Granadino et al. 2000b).

The biochemical and functional structure of FoxJ2 has been extensively studied. In addition to the DNAbinding domain, other functional domains, such as those involved in its nuclear localization or transcription activation, have already been characterized (Gómez-Ferreria and Rey-Campos 2003). Four regions of the molecule are essential for FoxJ2 to activate transcription: three transactivation domains, located on both sides of the Fork Head domain, and a proline/ glutamine-rich region; the latter appears to be required for the full activity of the other domains, although it lacks transactivation capacity by itself alone. The domains involved in nuclear translocation of FoxJ2 are the two regions flanking the Fork Head domain.

The FoxJ2 DNA-binding domain displays a dual DNA-binding specificity. This factor is able to recognize two different types of DNA sequences: type A-which shows a core element also found in DNA sequences recognized by other members of the Fork Head family-, and type B-which comprises a set of sequences different from type A, since they do not contain the core element. Previous reports have shown that FoxJ2 activates transcription of promoter regions containing any of these two types of sequences (Pérez-Sánchez et al. 2000a).

Although FoxJ2 is a well known transcription factor, its biological role has yet to be fully elucidated. Other members of this family develop their function by regulating the expression of several genes, with a direct impact on the transcription rate, as activators of transcription or/and as chromatinremodeling factors (Shim et al. 1998). Since FoxJ2 is widely expressed in the adult, it could be involved in a fairly general function of the organism. In addition, FoxJ2 may also be involved in early stages of development, since its expression starts immediately after activation of the zygotic genome (Granadino et al. 2000a, b).

In order to evaluate the biological function of FoxJ2, the main goal of this work, we used transgenic mouse technology. It is well established that the phenotype obtained by transgenic over-expression of a protein can yield evidence allowing us to speculate about its natural biological function. For this purpose, several FoxJ2 constructs were prepared and transgenic mice were generated. This article describes the analysis of these transgenic mice. The involvement of FoxJ2 in the regulation of cell adhesion molecule expression is also discussed.

\section{Materials and method}

PGK-FoxJ2 and PGK-GFP constructs

A fragment of the FoxJ2 cDNA encompassing just the CDS was cloned, under the control of the 
phosphoglycerate kinase 1 (PGK) promoter (McBurney et al. 1994), between the PstI and XbaI restriction sites of a PGK promoter plasmid vector. A short sequence encoding the HA epitope was cloned inframe at the $3^{\prime}$ end of the coding sequence. A SV40 poly-adenylation site was cloned downstream of the HA epitope. A similar construct, but with the cDNA for GFP from the pEGFP-N1 vector (Clontech), was used to generate GFP-transgenic control mice.

\section{Transgenic mouse generation}

Transgenic mice were generated using eggs collected from super-ovulated FVB female mice mated with FVB males. C57BL6J/6JxCBA/J crosses were used in the experiments involving in vitro culture of microinjected embryos. All related methods were as described in (Hogan et al. 1994; Andras et al. 2003). To identify transgenic founder animals, DNA was isolated from the tip of each mouse's tail, and screened by PCR amplification using primers: $5^{\prime}$ GGCATTCTGCACGCTTCAAAAG- $3^{\prime}$ and $5^{\prime}$-TT ATAGAGGTTGCGGAAGGACCAGC- $3^{\prime}$, which span the $3^{\prime}$-end of the PGK promoter: PCR amplifications were carried out using the following parameters: 35 cycles of $30 \mathrm{~s}$ at $94^{\circ} \mathrm{C}, 45 \mathrm{~s}$ at $56^{\circ} \mathrm{C}$, and $30 \mathrm{~s}$ at $72^{\circ} \mathrm{C}$. PCR-positive animals were further examined by Southern blot analysis using a DNA probe specific for the PGK-FoxJ2 construct.

Expression of recombinant fusion proteins

The FoxJ2 Fork Head domain was expressed in E. coli as a fusion protein with GST. A 320-base pair SmaI/ MunI restriction fragment of the FoxJ2 cDNA, coding residues ly37 to Ile142, was cloned in the SmaI and EcoRI restriction sites of the pGEX-3X vector (Amersham Pharmacia Biotech) in-frame with the GST open reading frame. Recombinant bacteria were grown overnight in Terrific broth (Ausubel et al. 1998), in the presence of $150 \mu \mathrm{g} / \mathrm{ml}$ ampicillin and $1 \%$ glucose, at $37^{\circ} \mathrm{C}$ with vigorous agitation. Cultures were centrifuged, and the bacteria were resuspended in fresh medium containing $150 \mu \mathrm{g} / \mathrm{ml}$ ampicillin and $0.5 \mathrm{mM}$ isopropyl-1-thiob-D-galactopyranoside and induced for $4-16 \mathrm{~h}$ at $37^{\circ} \mathrm{C}$ with agitation. Bacteria were centrifuged, resuspended in phosphate-buffered saline solution, and lysed in a French press. Triton $\mathrm{X}-100$ was added at $1 \%$, and the lysate was cleared by centrifugation. GST::FoxJ2 fusion protein was recovered from the supernatant by affinity chromatography on glutathione-agarose columns (Sigma). The protein was eluted overnight at $4^{\circ} \mathrm{C}$ with reduced $50 \mathrm{mM}$ glutathione in $250 \mathrm{mM}$ Tris- $\mathrm{HCl}, \mathrm{pH}$ 8.0. The purified GST::FoxJ2 fusion protein was dialyzed against $50 \mathrm{mM}$ Tris- $\mathrm{HCl}, \mathrm{pH} 8.0,2 \mathrm{mM}$ dithiothreitol, $15 \%$ glycerol, to remove the free glutathione used in the elution step, and used for EMSAs experiments.

DNA-binding assays

FoxJ2 binding to oligonucleotides corresponding to Connexin-43 and Cadherin-E promoter was assayed using standard EMSA procedures (Ausubel et al. 1998). Briefly, crude bacterial extracts or purified recombinant GST::FoxJ2 fusion proteins were incubated with radioactively labeled oligonucleotide probes in a buffer containing $10 \mathrm{mM}$ Tris- $\mathrm{HCl}, \mathrm{pH}$ 7.9, $50 \mathrm{mM} \mathrm{NaCl}, 2.5 \mathrm{mM} \mathrm{MgCl}_{2}, 1 \mathrm{mM}$ EDTA, $1 \mathrm{mM}$ dithiothreitol, and $4 \%$ glycerol. About $1 \mathrm{mg}$ of poly (dI-dC) zpoly (dI-dC) was added as a nonspecific competitor. Double-stranded oligonucleotide probes were labeled by filling the 59 protruding ends with [a-32P]dCTP using the Klenow fragment of DNA-polymerase I. Between 0.1 and $1 \mathrm{ng}$ of probe was used per assay.

\section{Transfections}

Cell transfection experiments were carried out using two cell lines: Hep3B human hepatoma, and C33 human cervix carcinoma. Cells were grown in either $1 \mathrm{~cm}$, or $3 \mathrm{~cm}$ diameter M24/M6 culture wells, and transfected using the FuGene reagent (Roche). A total amount of $1 \mathrm{mg} / \mathrm{M} 24$ well of DNA (10 mg/well for M6 Plate) was used in each transfection experiment. Luciferase activity was determined using the Single Luciferase assay kit from Promega. A constant amount of RSV- $\beta$ Gal plasmid was used to normalize the transfection efficiency.

\section{Results}

Low success rate of FoxJ2 transgenic mice

FoxJ2 transgenic mice were generated with a construct containing the cDNA of FoxJ2.L under the 
control of the PGK promoter (PGK-FoxJ2). We used the large isoform of FoxJ2 (FoxJ2.L) because it displays a higher transactivation activity compared to the short form (FoxJ2.S). The PGK promoter was selected because it is a well known ubiquitous and early expressed promoter; this promoter drives transgene expression from the earliest moment of embryonic development (Singer-Sam et al. 1992), an important feature since FoxJ2 expression starts at these stages.

The transgene construct also contained a sequence coding for the HA epitope, to make it easier to detect the transgenic protein. Previous experiments have shown that the HA epitope does not affect the functionality of the FoxJ2 protein (Pérez-Sánchez et al. 2000a, b). A similar construct containing a cDNA for the GFP-green fluorescent protein (PGKGFP), instead of FoxJ2, was used to generate control transgenic mice. The functional efficiency of these vectors (PGK-FoxJ2 and PGK-GFP) was previously confirmed by immunofluorescence and Westernblotting of cell lines transfected with both constructs (data not shown). Nine different FoxJ2 transgenic lines were generated and the litters were genotyped for the FoxJ2-transgene by PCR and Southern blotting (Fig. 1). In addition, GFP expression in the GFP-transgenic control mice was monitored by fluorescence (data not shown).

As summarized in Table 1, the live birth success rate obtained with the control transgenic mice was

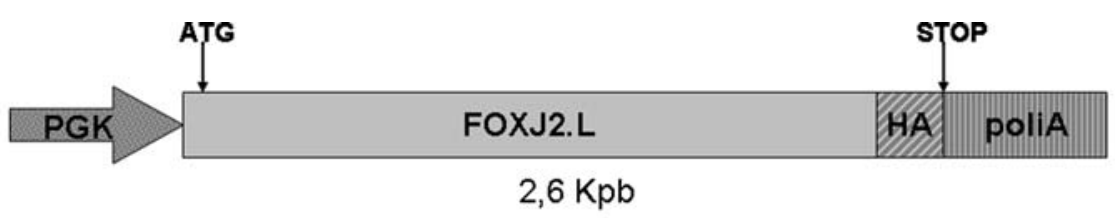

1

2 3

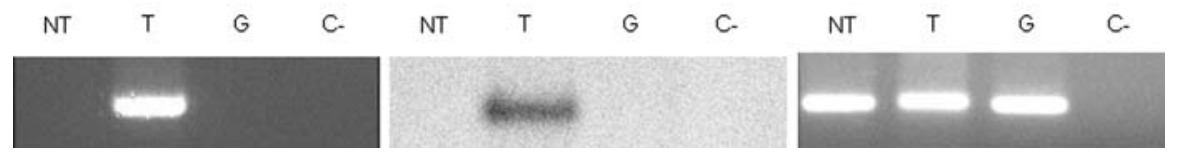

Fig. 1 FoxJ2 construct and genotyping. The figure shows the construct used in the transgenesis experiments. The full coding region of FoxJ2.L cDNA $(2.6 \mathrm{Kpb})$ was inserted immediately downstream from the PGK promoter. For reporting purposes, the HA (influenza virus hemaglutinin) epitope was cloned in-frame at the $3^{\prime}$-end of the FoxJ2L coding region. Finally, a SV40 virus polyadenylation signal was added at the $3^{\prime}$-end. The lower part of the figure shows the genotyping strategy used to identify the transgenic animals. The left panel shows a PCR experiment with genomic DNA from a potential transgenic animal, with primers specific of the transgene. The middle panel shows the Southern blot of the same experiment probed with a transgene-specific probe. Finally, the right panel shows a PCR-positive control with primers for an internal gene (NT) genomic DNA from a transgenic litter; (T) genomic DNA from a transgenic mouse; $(\mathrm{G})$ control genomic DNA; (C-) negative control, $\mathrm{H}_{2} \mathrm{O}$

Table 1 Transgenesis experiment results

\begin{tabular}{lcccc}
\hline DNA Construct & $\begin{array}{l}\text { Injected } \\
\text { oocytes }\end{array}$ & $\begin{array}{l}\text { Number survived } \\
(\% \text { of injected })\end{array}$ & $\begin{array}{l}\text { Number newborn } \\
(\% \text { of injected })\end{array}$ & $\begin{array}{l}\text { Number transgenic } \\
(\% \text { of injected })\end{array}$ \\
\hline Control & 422 & $332(75 \%)$ & $41(9,27 \%)$ & $11(4,47 \%)$ \\
PGK-GFP & 246 & $187(76 \%)$ & $25(10,16 \%)$ & $9(0,46 \%)$ \\
PGK-FOXJ2 & 1,922 & $1,423(74 \%)$ & $62(3,2 \%)$ & \\
\hline
\end{tabular}

The table shows the success rate of the FoxJ2-transgenesis experiments. Columns 3-5 shows the number of surviving embryos and animals and their percentage with respect to the total number of injected embryos (shown in column 2). Results are shown for FoxJ2 transgenic animals (PGK-FoxJ2), control GFP-transgenic animals (PGK-GFP), and control embryos mock-injected without DNA (Control). Differences observed were statistically significant at $P<0.05$. GFP-transgene expression was easily detected in by epifluorescence of the embryos or newborn animals (not shown) 
about $10.16 \%$ of the transferred embryos. The percentage of transgenic mice produced was $4.47 \%$. By contrast, these percentages fell dramatically in the case of FoxJ2 transgenic mice; where the percentage of live births was around $3.2 \%$, and in the FoxJ2genotyped transgenic mice, it was only about $0.45 \%$, which was significantly lower than expected $(P<0.05)($ Table 1$)$. As an additional control, nonmicroinjected embryos were also transferred to mice. Again, these experiments showed a much higher percentage of live births than in the case of FoxJ2 transgenic mice (Table 1).

However, although integration of the transgene was demonstrated for all FoxJ2 transgene mice, no transgene transcripts or protein were detected by RT-PCR and immunohistochemistry assays, respectively, in any of the transgenic mice analyzed (data not shown), except for two cases (see below). Since the activity of the FoxJ 2 construct had previously been checked by transfection assays, the failure of transgene expression detection suggests that most of the FoxJ2 transgenic mice that lived to adulthood did not actually express the transgene. Although the lack of transgene expression is not unusual in transgenesis experiments, the frequency of failure observed in FoxJ2-trangenic mice was much higher than expected. This might be explained by the reduced embryonic survival of transgenic embryos overexpressing FoxJ2.

Microinjected embryos over-expressing FoxJ2 show a blockage in early development

In view of the effect on survival rates, we considered it of interest to attempt to determine the stage of embryonic development at which over-expression of FoxJ2 could have a negative impact. Since the expression of the FoxJ2 gene starts very early in development (at the 8-cell stage), the negative effect of its over-expression could already be apparent at these early stages. To test this possibility, we monitored the development of microinjected embryos in in vitro cultures.

One-cell embryos were microinjected with the same overexpression constructs as were used in the transgenesis experiments described above and then kept in culture up to day 4 after injection. This is equivalent to the period of pre-implantational development. In these experiments we used circular plasmids instead of linearized plasmids. Embryos were microinjected with both PGK-FoxJ2 and PGKGFP constructs in combination. These allowed us to identify the embryos successfully microinjected and follow their development by live fluorescent microscopy, assuming that, since both constructs use the same kind of promoter, the embryos expressing GFP should also express FoxJ2. As a negative control, some embryos were microinjected with a PGK-Neo construct, a plasmid coding for an irrelevant gene (Neomycin-resistance gene) instead of the FoxJ2 plasmid. In these control experiments, PGK-GFP was also co-injected as a reporter of expression. In all cases, embryos were microinjected at the two pronuclear embryonic stages, from fertilized oocytes, and kept in culture until the blastocyst stage. As a further control, non-microinjected embryos were also cultured under the same conditions.

During the pre-implantation development in culture the surviving embryos microinjected with FoxJ2 (PGK-FoxJ2 + PGK-GFP) were compared with those microinjected with the control (PGK$\mathrm{Neo}+\mathrm{PGK}-\mathrm{GFP})$ and with those not microinjected (Fig. 2a). At E1.5, control- and FoxJ2-microinjected embryos, as well as non-microinjected embryos, displayed no differences in survival rates. Most of them developed to the 2-cell stage. Thus, neither the expression of FoxJ2, nor the microinjection itself caused severe problems to the embryo at these stages.

However, at a later stage, E2.5, a significant developmental blockage was observed in FoxJ2microinjected embryos $(P<0.05)$. These embryos did not develop beyond the 2-cell stage, while most of the control embryos were at the 3-4-cell stage. At E3.5 a higher percentage of the FoxJ2 microinjected embryos were still at an earlier stage of development, compared to the control embryos, which were already at the morula stage $(P<0.05)$. Moreover, at E4.5 the developmental blockage affecting embryos microinjected with the FoxJ2 construct was still apparent.

Therefore, these experiments showed that overexpression of FoxJ2 at early stages of development appears to induce a blockage in embryonic development approximately at E2.5. These data suggest that FoxJ2 over-expression has a negative impact on preimplantational development. 
A

DFoxj2+GFP ENeo+GFP DControl

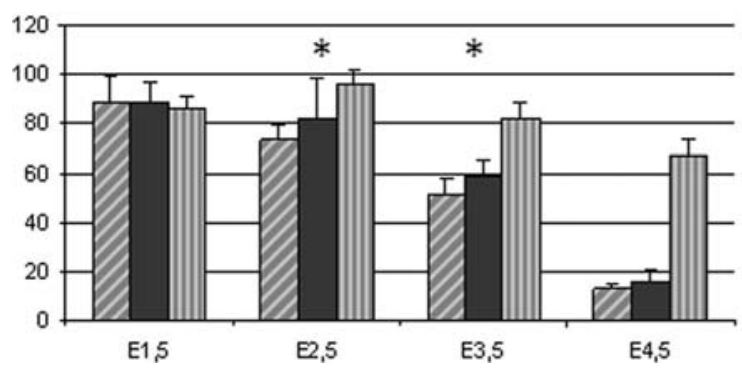

Fig. 2 FoxJ2 over-expression affects the early development of transgenic embryos. Panel (a) Pre-implantation development of double-injected FoxJ2 + GFP or Neo + GFP, embryos was followed in vitro until the 4th day after injection, when noninjected embryos reached blastocyst stage. The figure shows the percentage of surviving embryos at each stage of development from E1.5 to E4.5. FoxJ2 over-expression consistently induces a significant blockage in embryonic development approximately at E2.5 as compared to Neo + GFP control-injected embryos.

FoxJ2 over-expression shows a lethal effect on post-implantation development

To investigate further whether FoxJ2 over-expression also has an effect on post-implantational development, we performed a series of transient transgenesis experiments. Pseudopregnant mouse females were transferred with PGK-FoxJ2 or PGK-GFP microinjected embryos, and sacrificed on different days of gestation. The comparison of E8.5 and E9.5 embryos and deciduas from both transgenic mice revealed no differences in the predicted number of embryos. Morphology was also similar in both cases.

However, at E11.5 $(P<0.05)$ the survival rate of FoxJ2 transgenic embryos was lower than that of the control embryos (Fig. 2b); smaller deciduas and higher numbers of reabsorbed embryos were observed (Fig. 3). The same percentage of dead embryos and fetal reabsorption persisted at all subsequence stages of development.

Taken together, these data indicate that FoxJ2 overexpression has a lethal effect during embryonic development, starting around the E10.5 stage.

FoxJ2 over-expression induces heart hypertrophy in adult transgenic mice

As mentioned above, most FoxJ2 transgenic mice that live to adulthood did not express the transgene at

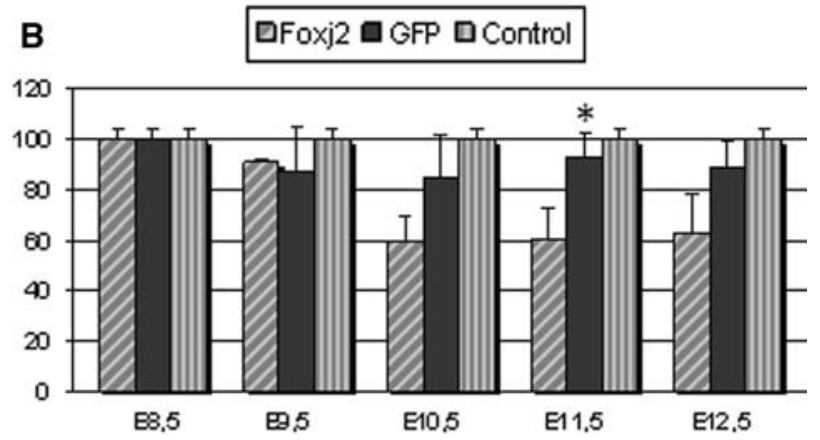

Panel (b) Post-implantation development of FoxJ2-transgenic mice was followed by transient transgenic experiments. Development was interrupted at different post-injection ages from E8.5 to E12.5. Embryos were collected from pregnant foster mothers and their developmental level was analyzed. From E10.5 stage the surviving FoxJ2-transgenic embryos dramatically decreased as compared to GFP-control transgenic embryos, or non-injected embryos. The asterisk indicates differences found to be statistically significant at $P<0.05$

the protein level. However, two adult transgenic animals (male and female) did show expression of the transgene. Immunohistochemistry and immunofluorescence experiments indicated mosaic expression of the transgene, with only some dispersed cells of organs and tissues expressing the Fox 2 transgene at the protein level (data not shown). This reduced and confined expression of the transgene might explain why these two transgenic mice were able to live to adulthood.

These two transgenic animals (from different litters) suddenly died at 9 (male) and 12 (female) weeks after birth. Post mortem analysis of these animals showed them to have died of cardio-respiratory failure. Both mice showed heart hypertrophy and massive lung hemorrhage with complete bronchoalveolar blood flooding; the heart hypertrophy was mainly along its longitudinal axis, as well as the top. The ventricles were enlarged and the heart-wall was swollen (Fig. 4a).

Although the female of these transgenic mice was fertile (two pregnancies), none of its progeny was transgenic. On the other hand, the male transgenic mouse was unable impregnate any females. This, together with their early death, made it impossible to found a colony from these transgenic lines.

These results also suggested that the male transgenic mouse could be infertile. Histological analysis of the animal's testes showed no mature spermatozoa 


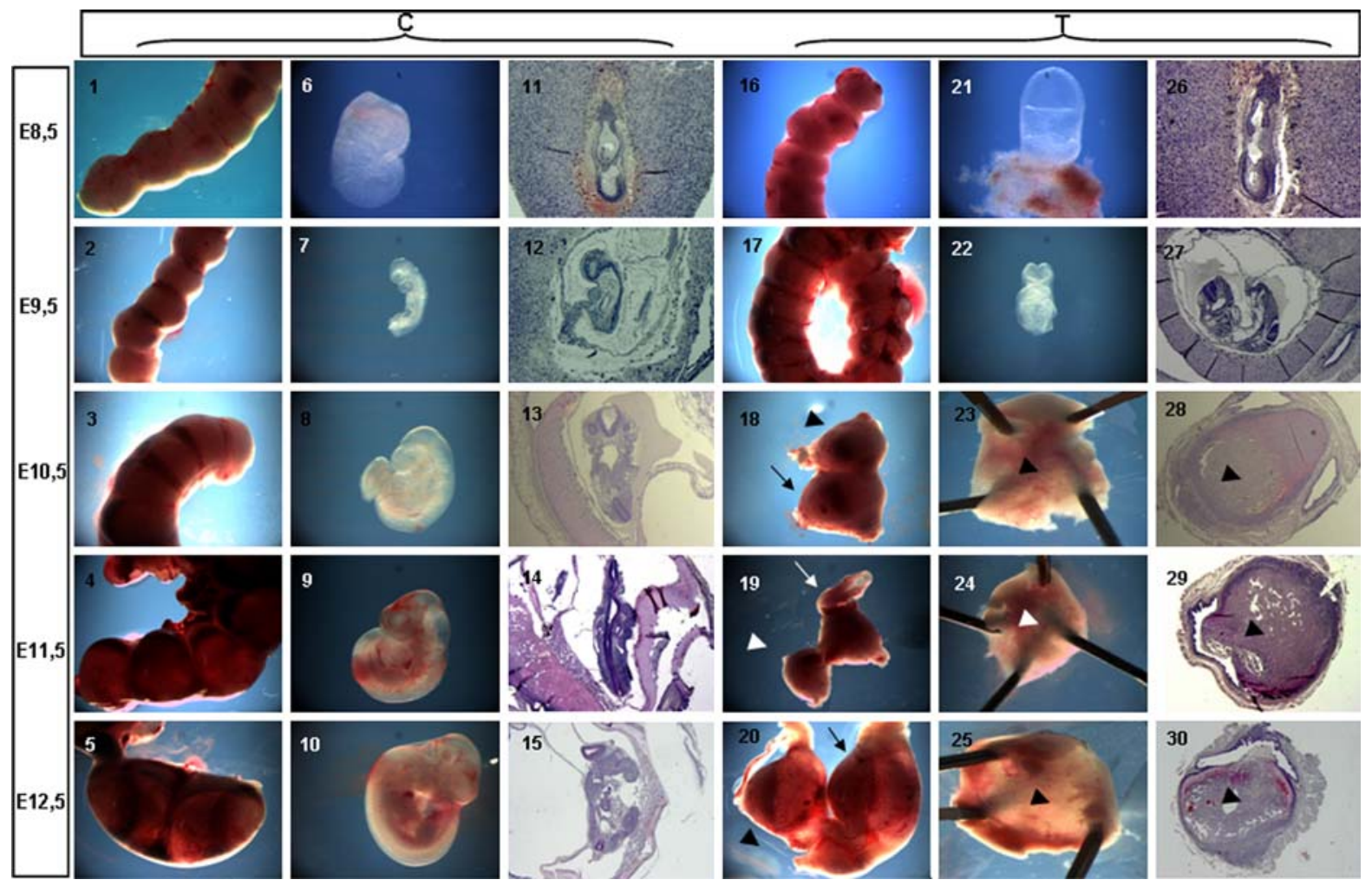

Fig. 3 Morphological analyses of FoxJ2-transgenic embryos at early post-implantation development. The panels show the macroscopic aspect and histological sections of deciduas and embryos from FoxJ2-transgenic embryos (T) and from control experiments (C) at different developmental stages from E8.5 to E12.5. As shown, embryonic death is observed in FoxJ2transgenic deciduas from E10.5 stage. These deciduas show signals of re-absorption of the dead embryos (indicated with an arrowhead). Panels 1-5 and 16-20 show uterine horns of control

in the seminiferous tubules, suggesting a failure of the spermatogenesis process. Condensate chromatin in round spermatids, which produces an arrest of meiosis events, and a narrow diameter of the seminiferous tubules, was also observed (Fig. 4b). This profile is considered a marker for sterility and probably explains the infertility of this animal.

In addition, the ovaries of the female FoxJ2 transgenic mice showed a high percentage of anomalies and reabsorption sites, consistent with the results obtained from the transient transgenesis experiments described above.

Taken together these data suggest that low level expression of the FoxJ2 protein, or high levels but with a mosaic distribution, may not have a lethal effect and could be compatible with life of transgenic and transgenic experiments, respectively, at stages E8.5 to E12.5. Panels 6-10 and 21-25, show the embryos extracted from these deciduas at same stages. Panels 11-15 and 26-30 show histological sections of these embryos inside the deciduas. Panels 18-20 show both a normal (shown with an arrow) and re-absorbed (shown with an arrowhead) deciduas. Panels 23-25 show the open deciduas with no embryos inside. Panels $28-30$ show histological sections of these deciduas with no embryos inside (the arrowhead shows the expected location for the embryos in both cases)

mice. However, it could have important effects on organs such as the heart, which finally lead to death.

\section{FoxJ2 candidate genes}

In order to identify the genes that may possibly be regulated by FoxJ2, we used a bio-informatics approach. The GeneBank sequences were scanned for FoxJ2-binding sites within promoter or enhancer regions. For this purpose, a matrix of frequencies for each nucleotide at each position of the sequences specific for FoxJ2 was constructed, and then used as a scanning probe using a computer program that we developed based on the Needelman and Wunch algorithm. This search yielded several candidate genes with promoter or/and enhancer sequences 

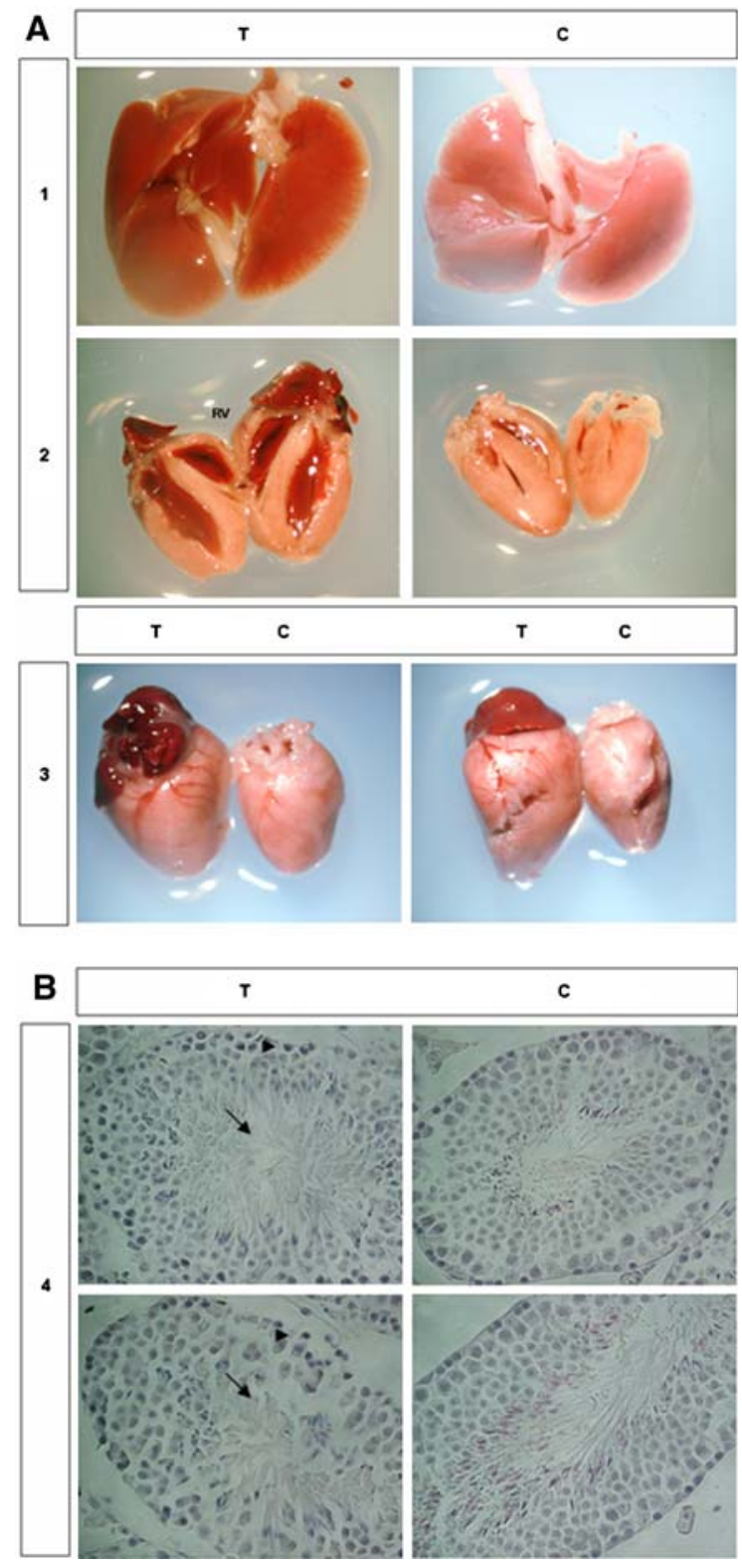

compatible with the FoxJ2-binding sequence. Among these, the gene coding for Connexin-43 (Cx-43) and the gene coding for E-Cadherin (E-Cad) had a high matching score; Cx-43 is a member of the family of proteins which form gap junctions, while the E-Cad protein is involved in cell-cell adhesion events.

To evaluate the ability of FoxJ2 to specifically bind to the promoter region of the genes coding for CX-43 or E-Cad, we performed EMSA assays with specific probes for these genes and GST::FoxJ2
\Fig. 4 Post-mortem analyses of surviving FoxJ2-transgenic mice. The figure shows the lung and heart of one of the two FoxJ2-transgenic mice, a male and a female that survived to adulthood and then died prematurely at 9 and 12 weeks, respectively. And histological sections of the testes from the male. (a) Post mortem analysis of these animals indicated that they died of cardio-respiratory failure. (1) The lung of the transgenic animal (T) shows a massive lung hemorrhage with complete broncho-alveolar blood flooding. (2) The heart of the transgenic showed a clear hypertrophy as compared to the control littermate (C). (3) This panel shows open views of the hearts, revealing that the heart hypertrophy was mainly along its longitudinal axis, as well as the top, and most notable on the right side of the heart. The ventricles (rv) were also oversized and the heart-wall was swollen. (b) Male FoxJ2transgenic mouse showed spermatogenesis impairment. (4) This panel shows histological sections of the FoxJ2-transgenic mouse testes. No mature spermatozoa were found within the seminiferous tubules (black arrows). Condensate chromatin in round spermatids (arrowhead), and a narrow diameter of the seminiferous tubules was observed, indicating an arrest of spermatogenesis at post meiotic stages

recombinant fusion protein (Fig. 5a). These experiments showed the formation of specific retarded bands when FoxJ 2 protein was incubated with $\mathrm{Cx}-43$ or E-Cad oligonucleotide probes demonstrating the ability of FoxJ2 to recognize and bind to the promoter regions of $\mathrm{Cx}-43$ and E-Cad. FoxJ2 protein did not bind to non-specific probes. As expected, the control GST protein alone did not bind to Cx-43 or E-Cad probes.

To evaluate whether the binding of FoxJ2 to the CX-43 and E-cad promoters results in transcription activation, we carried out a series of co-transfection experiments. We used FoxJ2 over-expression constructs, as an effector plasmid, together with Luciferase constructs driven by promoter regions of CX-43 and E-Cad, as reporter genes. As shown in (Fig. 5b), higher Luciferase activity (8-fold and 5-fold for Cx-43 and E-cad reporters, respectively) was observed in co-transfection experiments with FoxJ2 than in experiments with a control effector construct (PGK-Neo). Thus, our data indicate that the FoxJ2 protein is able to bind to $\mathrm{Cx}-43$ and E-cad promoters and transactivate their transcription.

These results suggest that the FoxJ2 transcription factor may be involved in the regulation of $\mathrm{Cx}-43$ and E-Cad expression. The similar expression pattern for these genes and that of FoxJ2, and the comparable phenotypes of the transgenic mice for FoxJ2 and those of Cx-43 and E-Cad, support this idea. 
Fig. 5 The promoter regions of Cadherin-E and Connexin-43 genes bind and respond transcriptionally to FoxJ2. (a) EMSA analyses with labeled oligonucleotide probes for the Cadherin-E and Connexin-43 promoter regions that show homology with consensus FoxJ2-

binding sites. As shown in lane 2 in both cases, recombinant FoxJ2 is able to form a specific complex when incubated with the probes. The formation of these complexes was competed efficiently with an excess of unlabeled probe (lane 3) and with a non-specific labeled probe (lane 4). (b) Transactivation of Connexin-43 and Cadherin-E promoter regions by FoxJ2. FoxJ2induced transactivation levels of the luciferase reporter gene under the control of Connexin-43 (right) or Cadherin-E (left) promoter regions in transient transfection experiments. As a positive control, we used a synthetic promoter with five tandemly repeated FoxJ2 consensus sites (PE2). The co-transfection of an expression vector of an irrelevant gene (Neo) with these reporter vectors served to determine their basal expression levels in the absence of added FoxJ2
A

A Conexina 43
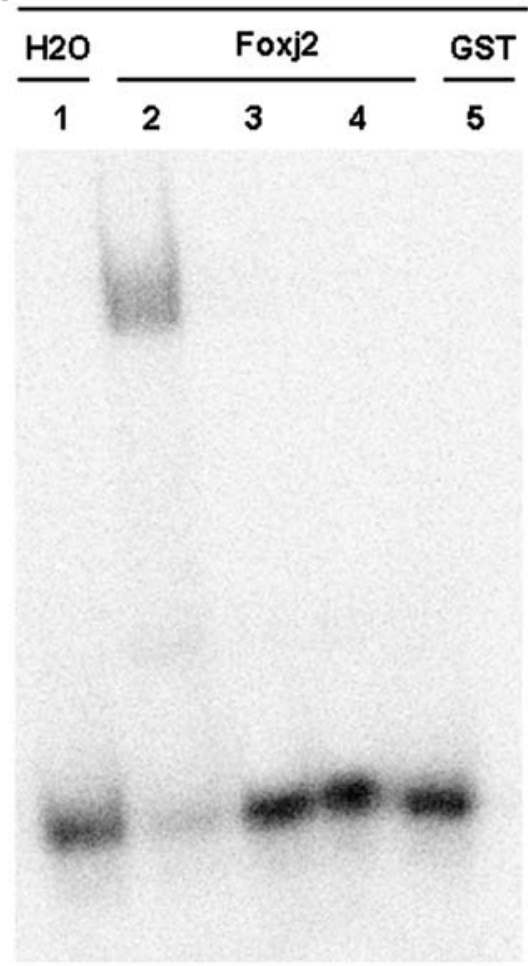

B

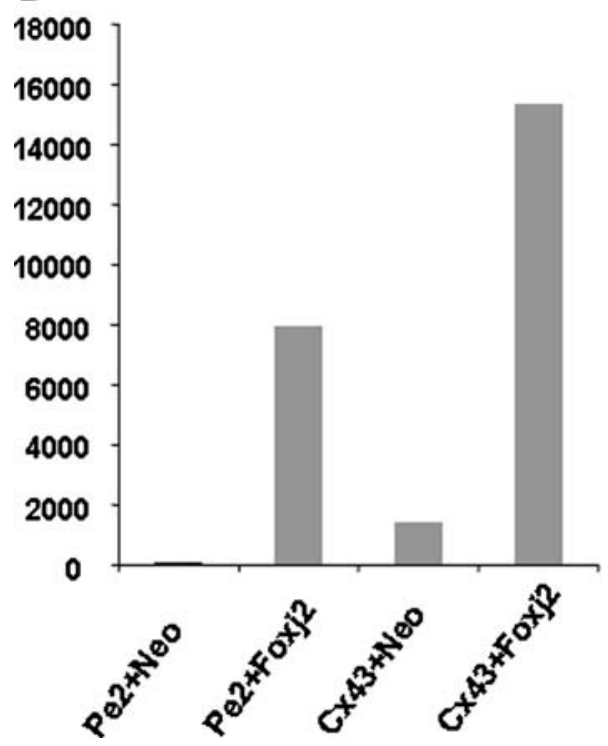

Cadherina E

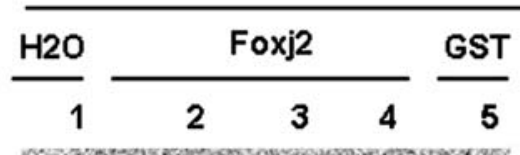

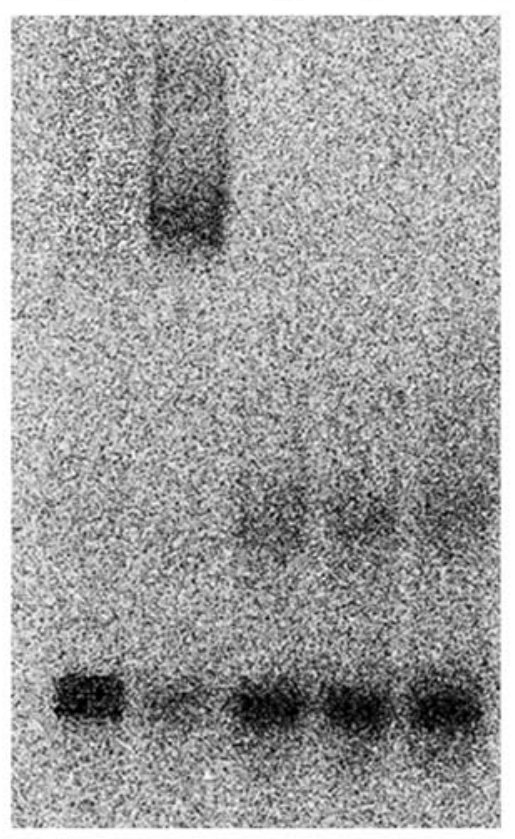

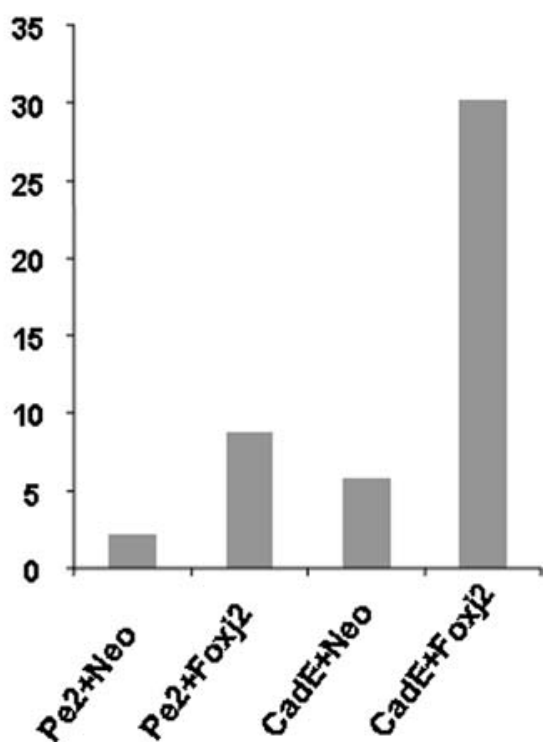

\section{Discussion}

In this study, we used a transgenic approach to the characterization of the biological function of FoxJ2. We have generated transgenic mice over-expressing
FoxJ2 as a means of unraveling the biological functions of this transcription factor. These studies indicated that live birth rate of transfer embryos overexpressing FoxJ2 was considerably lower than control transgenic transfer embryos. Furthermore, most 
of the transgenic animals generated did not show FoxJ2 transgene expression. Time-series analysis of transient transgenic animals revealed that the lethal effect of FoxJ 2 over-expression is already apparent as early as the E10.5 stage of embryonic development, producing abnormal embryos that are rapidly reabsorbed within the decidual tissue.

In vitro culture of early embryos over-expressing FoxJ2 transgene also showed a clear developmental blockage during pre-implantational stages. These studies showed that FoxJ2 over-expression may have a negative effect on development as early as E2.5. Since FoxJ2 expression starts soon after zygotic genome activation (it is already detectable at the 8-cell stage), our results suggest that its level of expression may need tight control in order to be compatible with embryo survival.

As mentioned above, our efforts to obtain adult transgenic mice overexpressing FoxJ2 only produced two animals out of a total of nine transgenic specimens. Moreover, these animals died soon (9-12 weeks) after birth. Postmortem analyses of these two animals showed common features that we may deduce were produced by the FoxJ2 overexpression, despite the small numbers involved. Thus, both animals showed heart hypertrophy and blood flooded lungs, which suggests that they died of a cardio-respiratory problem. These two animals were a male and a female. The male mouse showed abnormal testes histology. No mature spermatozoa were found within the seminiferous tubules, indicating a failure in spermatogenesis, which would result in sterility. This would explain why this animal was not able of impregnate any female mice. These observations are compatible with FoxJ2s having a role in spermatogenesis, since FoxJ2 expression is found at different stages of this process (Granadino et al. 2000a, b). Again, the precise levels of FoxJ2 expression appear to be essential for the development of its natural biological functions.

The next approach to characterizing the biological function of FoxJ2 was to search for genes regulated by this transcription factor. Database analysis of regulatory sequences (promoters and enhancers) for DNA-binding sites specific for FoxJ2 revealed several candidate genes. We choose two of them for further investigation, because they share the pattern of expression of FoxJ2 and were compatible with the phenotype of FoxJ2 transgenic mice. These two genes were those coding for the gap junction protein Connexin-43 and the cell-cell contact protein E-Cadherin, respectively. Both have FoxJ2-binding sites in their regulatory sequences. These sites are functional in binding to FoxJ2 in EMSA experiments. In addition, the promoter region of these two genes showed a response to FoxJ2 transactivation in transfection experiments. These data strongly suggest that $\mathrm{Cx}-43$ and E-Cad may be good candidates for regulation by FoxJ2. Furthermore, proteins of both genes display expression patterns similar to FoxJ2 (De Sousa et al. 1993; Collins and Fleming 1995) and, interestingly, the phenotypes of $\mathrm{Cx}-43$ and E-Cad mutant mice resemble those of our FoxJ2 transgenic animals. The knockout $\mathrm{Cx}-43$ has been reported to produce cardiac malformations (Ya et al. 1998; Reaume et al. 1995). Defects in the germ line and gonads (Juneja et al. 1999) and failure of spermatogenesis (Roscoe et al. 2001) were also observed in mice lacking $\mathrm{Cx}-43$. However, overexpression of $\mathrm{Cx}-43$ also produces similar heart defects (Ewart et al. 1997). In particular, the phenotype of our FoxJ2 transgenic mice more closely resembled the phenotype of $\mathrm{Cx}-43$ transgenic mice over-expressing Cx-43. Both exhibit heart defects involving ventricular hypertrophy, which results in decreased viability.

In addition, we favor up-regulation because overexpression is indeed the effect that we have recently observed in humans (Ramos et al. personal communication). In a different experimental system we have shown that a high level of FoxJ2 expression significantly correlates with $\mathrm{Cx}-43$ over-expression in gonad tissue. These results were obtained from infertile male patients with a severe spermatogenesis failure (Sánchez-Aparicio et al. personal communication).

Another reason to favor over-expression of the FoxJ2 targets is the fact that the transgenic mice expressing E-Cad also developed cardiomyopathy, with severely increased heart size (Luo et al. 2001; Ferreira-Cornwell et al. 2002).

It is tempting to speculate that the effects of overexpression of FoxJ2 that we observed could be mediated by a deregulation of the expression of genes such Cx-43 and E-Cad. This would point a potential role of FoxJ2 in cell-cell interactions, a phenomenon underlying many physiological process, such as spermatozoa maturation, heart muscle contraction, and also during embryonic development. 
Acknowledgements This work was supported by grants PM99-0103 and BMC2003-03763 from the Spanish Ministry of Education.

Open Access This article is distributed under the terms of the Creative Commons Attribution Noncommercial License which permits any noncommercial use, distribution, and reproduction in any medium, provided the original author(s) and source are credited.

\section{References}

Andras N et al (2003) Manipulating the mouse embryo, 3rd edn. Cold Spring Harbor Laboratoy Press, Plainview, NY, USA

Ausubel FA, Brent R, Kingston RE, Moore DD, Seidman JG, Smith JA, Struhl K (eds) (1998) Current protocols in molecular biology. John Wiley \& Sons, Inc., New York

Choi VM, Harland RM, Khokha MK (2006) Developmental expression of FoxJ1.2, FoxJ2, and FoxQ1 in Xenopus tropicalis. Gen Expr Patterns 6(5):443-447

Collins JE, Fleming TP (1995) Epithelial differentiation in the mouse preimplantation embryo: making adhesive cell contacts for the first time. Trends Biochem Sci 20(8):307-312

De Sousa P, Valdimarsson G et al (1993) Connexin trafficking and the control of gap junction assembly in mouse preimplantation embryos. Development 117(4):1355-1367

Ewart JL, Cohen MF, Meyer RA, Huang GY, Wessels A, Gourdie RG, Chin AJ, Park SM, Lazatin BO, Villabon S, Lo CW (1997) Heart and neural tube defects in transgenic mice overexpressing the Cx43 gap junction gene. Development 124(7):1281-1292

Ferreira-Cornwell MC, Luo Y et al (2002) Remodeling the intercalated disc leads to cardiomyopathy in mice misexpressing cadherins in the heart. J Cell Sci 115(8): 1623-1634

Gómez-Ferreria MA, Rey-Campos J (2003) Functional domains of FOXJ2. J Mol Biol 329:631-644

Granadino B, Pérez-Sánchez C, Rey-Campos J (2000a) Fork Head transcription factors. Curr Genom 1:353-382

Granadino B, Arias de-la-Fuente C, Pérez-Sánchez C, Párraga M, López-Fernández LA, del Mazo J, Rey-Campos J (2000b) Fhx (Foxj2) expresión is activated during spermatogenesis and very early in embryonic development. Mech Dev 97:157-160

Hogan B et al (1994) Manipulating the mouse embryo, 2nd edn. Cold Spring Harbor Laboratoy Press, Plainview, NY, USA

Juneja SC, Barr KJ et al (1999) Defects in the germ line and gonads of mice lacking connexin43. Biol Reprod 60(5):1263-1270

Kaestner KH, Silberg DG, Traber PG, Schutz G (1997) The mesenchymal winged helix transcription factor Fkh6 is required for the control of gastrointestinal proliferation and differentiation. Genes Dev 11:1583-1595

Kaufmann E, Knochel W (1996) Five years on the wings of fork head. Mech Dev 57:3-20
Kume T, Deng KY, Winfrey V, Gould DB, Walter MA, Hogan BL (1998) The forkhead/winged helix gene Mf1 is disrupted in the pleiotropic mouse mutation congenital hydrocephalus. Cell 93:985-996

Lin K, Dorman JB, Rodan A, Kenyon C (1997) Daf-16: an HNF-3/forkhead family member that can function to double the life-span of Caenorhabditis elegans. Science 278:1319-1322

Luo Y, Ferreira-Cornwell M et al (2001) Rescuing the Ncadherin knockout by cardiacspecific expression of $\mathrm{N}$ - or E-cadherin. Development 128(4):459-469

McBurney MW, Staines WA et al (1994) Murine PGK-1 promoter drives widespread but not uniform expression in transgenic mice. Dev Dyn 200(4):278-293

Ogg S, Paradis S, Gottlieb S, Patterson GI, Lee L, Tissenbaun HA, Ruvkun G (1997) The fork head transcription factor DAF-16 transduces insulin-like metabolic and longevity signals in C. elegans. Nature 389:994-999

Pérez-Sánchez C, Gómez-Ferrería MA, de la Fuente CA, Granadino B, Velasco G, Esteban-Gamboa A, Rey-Campos J (2000a) FHX, a novel fork head factor with a dual DNA binding specificity. J Biol Chem 275:12909-12916

Pérez-Sánchez C, Arias-de-la-Fuente C, Gómez-Ferrería MA, Granadino B, Reycampos J (2000b) FHX.L and FHX.S, two isoforms of the human fork-head factor FHX (FOXJ2) with differential activity. J Mol Biol 301: 795-806

Reaume AG, de Sousa PA et al (1995) Cardiac malformation lacking connexin43. Science 267(5205):1831-1834

Roscoe WA, Barr KJ et al (2001) Failure of spermatogenesis in mice lacking connexin43. Biol Reprod 65(3):829-838

Shim EY, Woodcock C, Zaret KS (1998) Nucleosome positioning by the winged helix transcription factor HNF3. Genes Dev 12:5-10

Singer-Sam J, Chapman V et al (1992) Parental imprinting studied by allele-specific primer extension after PCR: paternal X chromosome-linked genes are transcribed prior to preferential paternal $\mathrm{X}$ chromosome inactivation. Proc Natl Acad Sci USA 89(21):10469-10473

Tan PB, Lackner MR, Kim SK (1998) MAP kinase signalling specificity mediated by the LIN-1 Ets/LIN-31 WH transcription factor complex during $C$. elegans vulval induction. Cell 93:569-580

Weigel D, Jurgens G, Kuttner F, Seifert E, Jackle H (1989) The homeotic gene fork head encodes a nuclear protein and is expressed in the terminal regions of the Drosophila embryo. Cell 57:645-658

Wijchers PJ, Hoekman MF, Burbach JP, Smidt MP (2006) Identification of forkhead transcription factors in cortical and dopaminergic areas of the adult murine brain. Brain Res 1068(1):23-33

Ya J, Erdtsieck-Ernste EBHW, de Boer PAJ, van Kempen MJA, Jongsma H, Gros D, Mooorman AFM, Lamers WH (1998) Heart defects in Connexin43-deficient mice. Circ Res 82:360-366

Zhou S, Zawel L, Lengauer C, Kinzler KW, Vogelstein B (1998) Characterization of human FAST-1, a TGF beta and activin signal transducer. Mol Cell 2:121-127 